\title{
Research on Media Innovation System Based on Mobile Augmented Reality Technology
}

\author{
Chen Zhen ${ }^{\mathrm{a}, *}$, Gao Wei, Zhong Zhi \\ School of Fusion Media, Sichuan University of Media and Communications, Chengdu, Sichuan, 611745, \\ China \\ a email: chenzhen.speak@163.com \\ *corresponding author
}

Keywords: Augmented Reality, Media Systems, Innovative Research

\begin{abstract}
Augmented reality is an extension of virtual reality, and the technical operation of augmented reality is mainly to integrate the actual situation around the user and the virtual scene calculated by the computer organically, so as to enhance the user's further cognition of the real world. Augmented reality can make life scenes more realistic, using the human senses of touch, vision and hearing to interact with each other, enabling customers to interact naturally with the environment, mostly in the fields of education, health, entertainment, industry and culture. Media fusion is an entry point in which the media system is built with the help of some smart phone hardware devices.
\end{abstract}

\section{Introduction}

Under the current social development background, the concept of virtual reality is becoming popular in the world, and has become a hot topic that industry and capital market attach great importance to and pursue. Both at home and abroad, there is a positive layout. Under the premise of satisfying the practicability, if we can attach importance to the offline experience activities, and pay attention to the offline experience interaction, design the solution with low cost, simple, convenient copy and simple operation, we can show the good effect, and enhance the participation of the users, and also have a certain industry promotion value, which can be well applied in the media culture, advertising and other industries.

In this paper, the research on the current technology, mobile augmented reality of the establishment and implementation of the media system. Mobile augmented reality's melt-in-themedia technology is embodied in the mobile-side app application, which covers four word systems, all requiring users to use the smartphone's camera for picture scanning, as a means of performing different forms of display enhancement experience, enabling users to get a better sense of experience with the help of sound, pictures and gestures.

\section{Key Technologies}

\subsection{Image Matching and Recognition}

Image matching and recognition technology is a prerequisite for mobile display enhanced melt media technology, and image matching technology is also very important for this technology, in which matching refers to finding the characteristic correspondence between two graphs of the same scene. There are four key steps, which are to detect the feature points, to describe the vector according to the extracted feature points, and then to roughly match the feature points, and finally to eliminate the wrong feature points. where the approximate flow of algorithm execution is to first calculate the detection and description of any feature point, the technology adopted is ORB feature extraction, then use the Euclidean distance of the feature point to complete the rough matching between the images, and finally use the RANSAC counting algorithm to screen these rough matching points to complete the final matching. the approximate flow is shown in figure 1. 


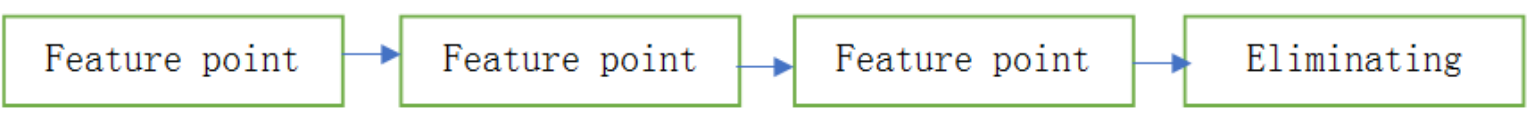

Figure 1 Image feature matching

\subsection{D Registration}

3d registration technology refers to the real-time tracking of the camera's pose position information, and finally finds the correct position of the virtual information in the real scene. finally, the virtual information and the real scene are superimposed and displayed in real time on the display. this technology can help realize the seamless fusion of real and virtual scenes. the current common technologies include camera calibration registration and affine transformation registration.

\subsection{The Combination of Reality and Reality}

After image matching recognition and 3D registration, we get enough information to set the virtual model position, and then combine the real scene with the virtual information, and finally present it in front of the customer, which is the technology of the combination of virtual reality. in order to help achieve the effect of real narrative seamless combination, virtual objects also need to automatically adjust the texture information according to the surrounding environment and the changes of light to achieve the occlusion of the object by light and the change of light and shadow to realize the immersion of the real environment.

\subsection{Interactive Control}

Interactive control technology is closely linked to real technology and tracking registration technology in mobile augmented reality system. Interactive control technology can directly influence the interactive experience of virtual object and real world. In AR system, humancomputer interaction has carried out a lot of application development and research work under the requirement of input and output of computer, but at present, the human-computer interaction requirement of AR system can not be satisfied only with the current hardware devices such as keyboard and mouse operating system. Such as gestures, speech recognition and animation[1].

\section{The Main Functions of a Reality-Enhanced Media System}

\subsection{Augmented reality function}

Users can use the mobile smartphone's camera to scan and recognize the card, and when the camera captures images of the same feature points stored in the database, it can match the corresponding three-dimensional model and video, so that it can be displayed in the screen, and the augmented reality effect can be realized.

\subsection{Gesture interaction}

In order to enhance the interactive function of AR system and the entertainment of the system, the gesture control function of the model is developed, when scanning the recognition picture, the customer can find the corresponding three-dimensional model in the virtual space, while the user can use the mobile phone touch screen to control the corresponding model gesture, so as to communicate and interact with the model. Clicking is the user's one-handed click on the model, the model will produce animation and sound, in addition to more close to the user's life habits, will add some colliders to the model, when the user clicks on the model, there will be a click effect, rather than let the user feel that they are just clicking on the screen. The operation of zooming is that the user uses two fingers to slide to control the scaling of the model, the whole scale can be set to 0.5 times to three times, in order to produce a better experience effect, the zoom effect on the whole screen, not just the model. Rotation is the rotation of a 360-degree control model in the horizontal direction by using a single finger to slide on the screen. The mobile function requires the user to use two fingers to operate in the same direction at the same time to control the model to follow the 
finger to move.

\subsection{Particle effects}

Particle effects are mainly applied to the main interface of the UI, and their significance is to improve the fidelity of the effect.

\subsection{Voice animation}

In the voice animation module, the main use is the Unity3D embedded Animation animation system, through the state of animation to transform and interactive control.

\subsection{Color mapping}

The effect is to identify the two-dimensional code to the real-time image of the threedimensional model, the two-dimensional image can be colored, and finally reflected in the threedimensional model. In order to achieve this effect, two algorithms of image processing, namely texture mapping and bilinear interpolation, are mainly used.

\subsection{QR code identification}

Using augmented reality for two-dimensional code recognition, displaying the recognition on the phone's screen will have a better recognition effect[2].

\section{Dissemination and use of the System}

In the augmented reality system, the use of the use will appear related scenes, such as AR model, video, QR code scanning and so on, in which the AR model scene is mainly to use the mobile phone camera to recognize the picture, after the recognition is completed, it can be rendered and superimposed in real time in the video, and the size and location of the model can be controlled by gesture operation. as shown in Figure 2. The video scene is image recognition, after the recognition can be superimposed a video and recognition of the size of the video to play. as shown in Figure 3. The two-dimensional code scan scene refers to when the camera scans the two-dimensional code, the blue box appears on the top of the two-dimensional code said twice, the final content can be displayed.

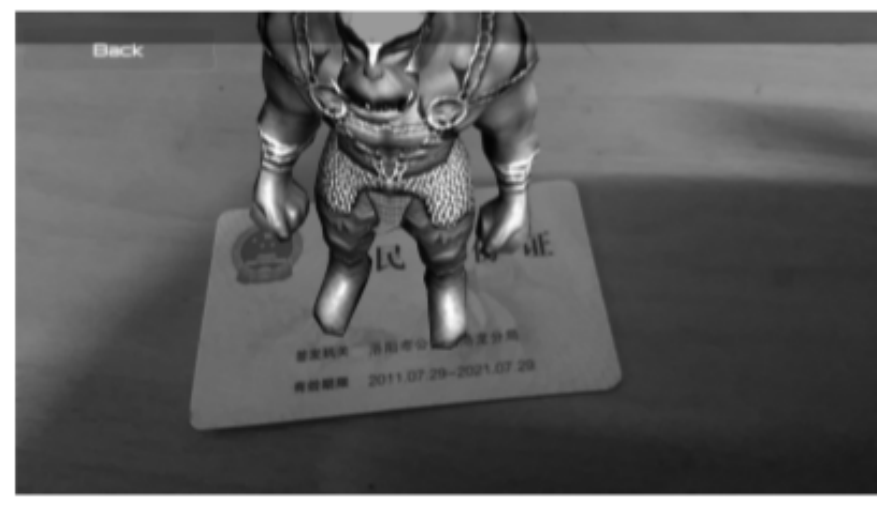

Figure 2 AR Model Scene 


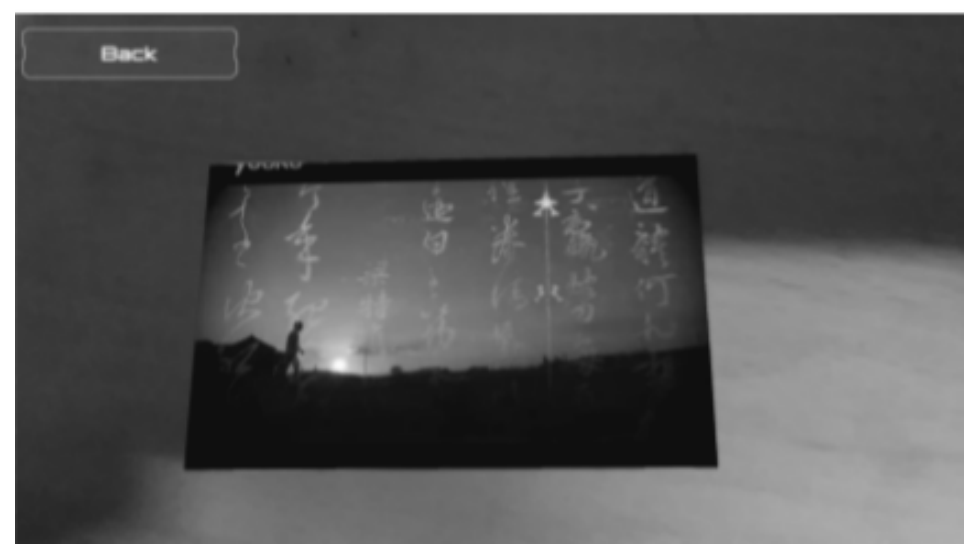

Figure 3 AR Video Scene

\section{Future Development of Media Augmented Reality Technology}

With the popularization of high-definition digital camera equipment, the way of obtaining video information is becoming more and more simple, people can use a variety of tools to obtain digital video, and with the development of digital media and the deepening of media technology, the demand for special effects application of digital will become more and more in the future. These market demands have promoted the development of animation and video interaction technology, and these developments will also promote the development and popularization of media augmented reality technology. For example, film, based on the rapid development of digital technology, can integrate real-time video as a technology to create immersive film, this film viewing mode will directly promote the essential transformation of the artistic form of film. And the media augmented reality technology will make the role substitution of the electronic live broadcast can be realized, and when the media augmented reality technology is sufficient, it can make the temporary replacement of the target in the event of failure, so as to ensure the smooth progress of the live broadcast.[3].

\section{Conclusion}

For the related innovation of the augmented reality system of melt media, the technology mainly uses Unity3D as the development engine, which can basically realize the effect of augmented reality. Its main application way is to use the mobile media to install the corresponding app, and to obtain the effect of augmented reality by using the mobile device for image recognition and scanning. And through the combination of mobile augmented reality technology and Unity3D, the design of melt media system can develop app with various scene uses, enrich and upgrade the scene creation and content display, and add the voice interaction function, which can make the application of app more convenient and effective, and further advance and strengthen the application of augmented reality technology in other fields.

\section{References}

[1] Pan Shangshi. Augmented reality and implementation of QR code content recognition. Single Chip Microcomputer and Embedded Systems Applications, vol. 20, no. 01, pp. 24-26+34, 2020.

[2] Zhao Hailiang. Research and design of augmented reality applications based on mobile terminals. Electronic World, no. 24, pp. 175-176, 2019.

[3] Zhang Fulu. Research on the construction of TV melt media interactive system. Western Radio Television, no. 24, pp. 31-32, 2019. 